\title{
Gender Differences in Computer-mediated Communication
}

\author{
Liping Fan \\ School of Foreign Language, Shandong Jiaotong University, Jinan, 250357, China \\ 365721640@qq.com
}

Keywords: gender, language communication, Computer-mediated Communication.

\begin{abstract}
Traditionally it is widely acknowledged that there are gender differences in using language. Nowadays with the development of network, computer as a communication medium has attracted users around the world due to its convenience and efficiency. The problem is proposed whether gender differences in the real-world communication exist in the computer-mediated communication. Through analyzing exchanging messages from QQ, it is found that the gender-specific communication does exist in computer-mediated communication. And then reasons are explored for the existence of the phenomenon.
\end{abstract}

\section{Introduction}

With the approaching of Information Age, Internet has been largely praised as a tool that allows people from around the world to communicate, for example, by qq, an instant messaging software services developed by the Chinese company Tencent Holdings Limited. Also, it is praised that the Internet provides a gender-less, age-less, race-less and any-other-bias - less opportunity for interaction[3]. Communication which is mediated by the computer and the Internet may be described with a number of terms, including virtual communication, online communication, electronic communication, cyber communication, or even cyber conversation...etc. All these are technically referred to as Computer-mediated Communication (CMC), which can simply be defined as a domain of information exchange via the computer (Baron, 1998)[1].

In the real world, many researches have been made into the relationship between language and gender. It is widely accepted that there are gender discrepancies in using language. Men tend to use language to negotiate status while women to establish intimate relationships with others.

Now are these traditional gender roles in real-world communication behavior perpetuated in computer-mediated communication? This paper will explore this problem by analyzing instant messages from qq.

\section{Language and gender}

According to Tannen [5], "communication isn't as simple as saying what you mean. How you say what you mean is crucial, and differs from one person to the next, because using language is a learned behavior: how we talk and listen are deeply influenced by cultural expectations". Women and men are like people who have grown up in two subcultures - they have two broad different styles of speaking and establishing social status.

In the process of socializing with peers, children generally tend to play with other children of the same gender, resulting in different ways of creating rapport and negotiating status within their group: childhood play is where much of our conversational style is learned [5].

The main distinction between the way boys and girls communicate is that girls generally use the language to negotiate closeness - that is, to establish intimacy as a basis of friendship (collaborationoriented); and, in comparison, boys generally use language to negotiate their status in the group (competition-oriented).

The theme of using power to negotiate status by males and cooperation to establish rapport by females is consistently played out throughout adulthood and repeated in the social and linguistic communicative styles between the two sexes at all levels: at home, work, meetings, social occasions, 
and in personal, casual and formal contacts. Consequently women and men tend to have different habitual ways of saying what they mean.

\section{Gender Differences in Computer-mediated Communication}

To answer the question whether the gender differences in real-world communication will be carried on into computer-mediated communication, the paper will analyse data collected from chatting records of men and women in QQ, the most popular chatting software in China. Analysis will be made from three aspects, that is, topic choice, emoticon and greeting.

In terms of topic choice, male users in QQ tend to talk more about technology-related topics such as cars or computers while female conversations focus on emotional subject matters. Male conversation generally deals with what is seen as more traditionally male topics, such as cars, computers, video games, and girls. Of the topics that appeared in male exchanges, $22 \%$ related to cars, $25 \%$ to computers, $26 \%$ to video games, and $15 \%$ to the opposite gender. Other topics may include what's happening in business, the stock market, the sports, or politics. Men do gossip (although they may not call it that) in the sense of talking about themselves and other people. But they tend to talk about political rather than personal relationships: institutional power, advancement and decline, a proposal that may or may not get through the committee, a plan for making money. When men do talk about their wives and families, the talk is liable to be brief, not belabored and elaborated in depth or detail.

Female users in QQ, on the other hand, tend to choose topics of a different nature. Emotional support is frequently sought and given, conversations revolve around common interests, and if males are discussed, they tend to be fictional. Within the female exchanges, a full $50 \%$ of the topics concerned matters of emotional support, $20 \%$ were of males (15\% fictional or famous men, $5 \%$ real) and the rest related to common interests.

This discrepancy in content resembles real world interaction as well. Lynn Smith-Lovin and Dawn Robinson[4] note about real space discussion that "women's talk is generally oriented to maintaining relationships and developing intimacy" and that "men are taught to be more instrumental, whereas women are taught to be more socioemotional, supportive". Therefore it is safe to conclude that whatever the medium of communication is, men and women still follow certain traditional conversational patterns.

With regard to the use of emoticons, QQ users use them to express their personal feelings. The use of emoticons also differs greatly between male and female conversations. In an average 50 conversations, there will be approximately 32 emoticons if the QQ users are female, but only 9 if the users are male. Among the conversation records, emoticons are the most frequently used to convey laughs or smiles, such as $\left.{ }^{\wedge} \wedge \wedge,{ }^{\wedge}{ }^{\wedge},:-\right)$, etc. It seems that smile is the most popular expression QQ users like to use. Actually, what this really implies is QQ users' concern for the talk to go on well. The functions of smiling emoticons are often the same as those of laughs or even minimal responses: to show their good will and support to others. The high frequency of emoticons use among female QQ users than male ones also indicates that women are more supportive while men are instrumental in conversations, congruent with real space interaction.

With respect to greetings and farewells, which play an important social role in QQ interaction, men and women also display different preference. Males demonstrate a tendency to ignore greetings and goodbyes in their conversations, starting and finishing their talk abruptly. Females on the other hand will generally open a conversation with a greeting of some sort, and when they need to end the conversation, they always state their reason for leaving before saying goodbye. In 50 different QQ messaging exchanges between a pair of males, the conversation was opened with a greeting 12 times and ended with a goodbye 16 times. In 50 MSN messaging exchanges between a pair of females, the conversation started off with a greeting 34 times, and finished with a goodbye 36 times.

This may be relevant to the differences in tone between the sexes; to formally open and close a conversation is to be polite, a characteristic more likely to be found in the gentler female conversations as opposed to the rougher male ones. This finding corresponds to Lakoff's observation, which states that women talk more politely. 
Taken the above as whole, it is clear that just like in face-to-face environments, gender related stereotypical patterns do exist in computer-mediated communication.

\section{The reason for the gender difference in computer-mediated communication}

One of the features of virtual communication is that participants in virtual interactions are often anonymous or partially anonymous. Kiesler [4] elaborates on the impact anonymity can have on communication: when communication lacks dynamic personal information, people focus their attention on the message rather than on each other. Communicators feel a greater sense of anonymity and detect less individuality in others than they do talking on the phone or face-to-face. They feel less empathy, less guilt, less concern over how they compare with others, and are less influenced by norms.

Kiesler further explains that the receivers in CMC constitute an easily accessible audience that is in fact a "social hodgepodge" and "the only clue the sender has to the receiver's identity and situation may be his or her name and writing style". Other commonly used indications of the receiver's status, gender, race, and appearance, are missing.

Given what Kiesler illustrated, virtual communication should be gender free, which is quite the opposite of the results of above studies. What is the reason?

The reason, it seems, lies very much in the different approach taken by women and men towards this new electronic technology - an approach which is congruent with the socialization and integration of males and females into society.

Men apparently see the opportunity provided by this technology as a chance to further one's own influence, by gaining valuable information and by extending one's own authority and respect in society; while women ostensibly view this technology as an opportunity to nurture existing relationships and develop new ones. Language styles online are different, to a certain extent, because they reflect the different goals of the users.

\section{Conclusion}

Based on the data analysis from QQ messages, it can be concluded that gender differences do exist in computer-mediated communication, resembling those in real world exchanges. With regard to the reason, it is claimed that male and female try to extend their different roles in real world to the virtual world.

\section{References}

[1]. Baron, N. S. Letters by phone or speech by other means: The linguistics of e-mail. Language and Communications, 18(2), 1998, pp.133-170.

[2]. Kiesler, S. The Hidden Message in Computer Networks. Harvard Business Review, 64(1),1986, p46-58.

[3]. Shea, V. Net Etiquette, San Francisco, USA: Albion Books. 1994

[4]. Smith-Lovin, M. \& D. T. Robinson. 1992. Gender and conversational dynamics. In Ridgeway, C. (Ed.), Gender, Interaction, and Inequality. pp. 120-146. New York: Springer-Verlag.

[5]. Tannen, D. The Power of Talk: Who Gets Heard and Why, article from Harvard Business Review, September, v. 73, n5,1995, p 138-148 